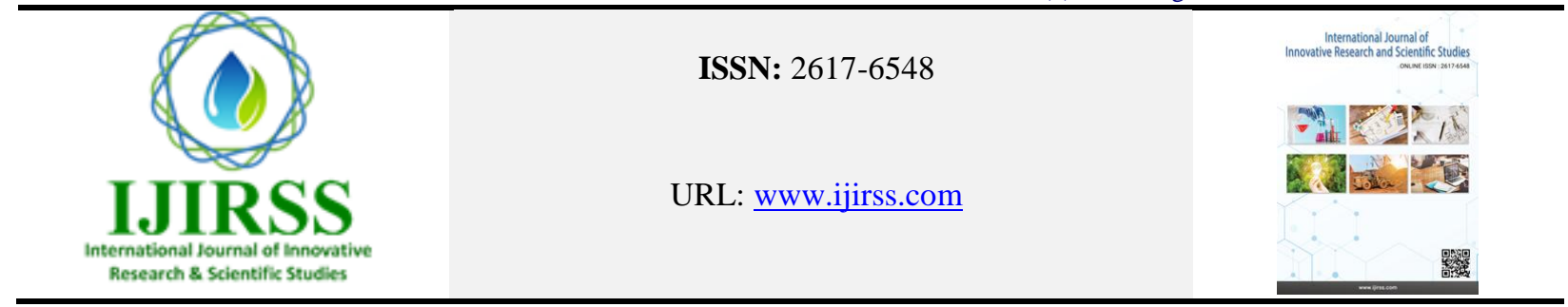

\title{
Emulsion Types, Stability Mechanisms and Rheology: A Review
}

\author{
Sweeta Akbari ${ }^{*}$, Abdurahman Hamid Nour ${ }^{2}$ \\ 1,2 Faculty of Chemical \& Natural Resources Engineering, Universiti Malaysia Pahang, Lebuhraya Tun Razak, 26300 Gambang, \\ Pahang, Malaysia \\ *Corresponding author: Sweeta Akbari (sweeta.akbary11@yahoo.com)
}

\begin{abstract}
Emulsions have been widely used in different industrial processes. The development and production of good quality emulsions depend on the knowledge of emulsion preparation, stability mechanisms and rheological studies. To form stable emulsions, an emulsifier is required to reduce the droplet sizes of the emulsions and enhance the emulsion stability. The purpose of this review article is to provide information about types of emulsions, stability mechanisms and rheological studies as well as factor affecting the stability of emulsions.
\end{abstract}

Keywords: Emulsion, Emulsion type, Stability mechanism, Viscosity, Droplet size.

DOI: 10.53894 /ijirss.v1i1.4

Funding: This study received no specific financial support.

History: Received: 12 January 2018/Revised: 2 March 2018/Accepted: 15 March 2018/Published: 21 September 2018

Licensed: This work is licensed under a Creative Commons Attribution 4.0 License $($ (c) EY

Acknowledgement: Both authors contributed to the conception and design of the study.

Competing Interests: The authors declare that they have no conflict of interests.

Transparency: The authors confirm that the manuscript is an honest, accurate, and transparent account of the study was reported; that no vital features of the study have been omitted; and that any discrepancies from the study as planned have been explained.

Ethical: This study follows all ethical practices during writing.

\section{Introduction}

An emulsion is a mixture of two immiscible liquid phases where one phase is dispersed into another. Basically, an emulsion is consisting of a continuous phase which is known as external phase, where the droplets are dispersed, and a dispersed phase which is defined as internal or discontinuous phase[1]. Emulsions are found in different industries such as food, pharmaceutical, agricultural, cosmetics, and petroleum [2]. An emulsion is not contained only water and oil; it may also contain some solid particles and even gas. Inherently, an emulsion is an unstable system due to the unfavorable contact between oil and water phases. Although, some emulsions are stable as a result of the small drop sizes and the existence of interfacial film, which surrounds the drops [1,3,4]. To form an emulsion a mechanical force is required to disperse one phase into another. However, the emulsions formed without adding any surface active material would not be stable, and the emulsion phases will start to separate in different layers based on their density differences. Therefore, addition of surfactants are necessary to make a stable emulsion for a period of long time.

To understand more about the emulsions, this review is focused on the study of emulsions types, stability mechanisms and rheological studies. 


\section{Types of Emulsions}

Essentially, there are three types of emulsions which are water-in-oil (W/O), oil-in-water $(\mathrm{O} / \mathrm{W})$, and complex emulsions such as water-in-oil-in-water $(\mathrm{W} / \mathrm{O} / \mathrm{W})$. The complex emulsion also known as a multiple emulsion [5]. The three types of emulsion are displayed in Figure 1.

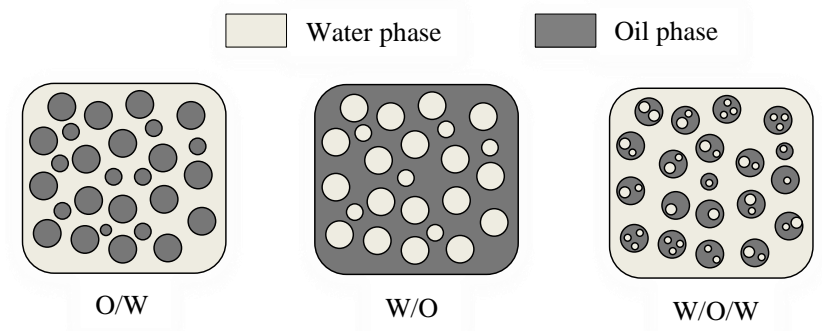

Figure-1.

Classification of oil emulsions.

Typically, if the oil is dispersed phase, the emulsion is named $\mathrm{O} / \mathrm{W}$, but if the aqueous medium is the dispersed phase, the emulsion is named W/O [6]. On the other hand, the multiple emulsions are complex systems. They can be considered as emulsions of emulsions.

\subsection{Water-In-Oil Emulsions (W/O)}

A water-in-oil emulsion is the type of emulsions which the continuous phase is usually hydrophobic materials such as oil and the dispersed phase is water [7]. More than $95 \%$ of the crude oil emulsion formed in the oil field are the W/O type [8]. The W/O emulsions contain three substances such as; a solvent, a surfactant, and water as demonstrated in Figure 2.

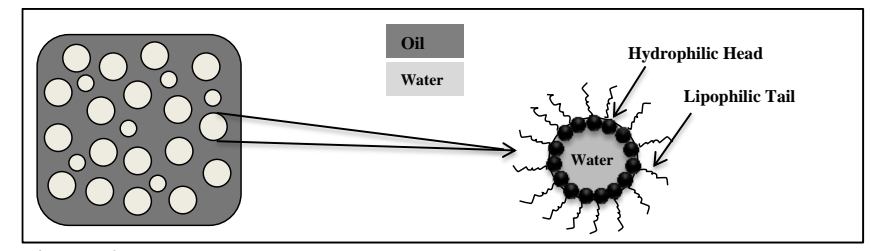

Figure-2.

W/O emulsion and emulsifiers on the surface of the dispersed phase (Water).

These compositions play a very important role in the formation of W/O emulsions [9]. Several studies have shown that the stability is the most important characteristic in W/O emulsion and generally these emulsions stabilized with natural surfactants such as resin and asphaltenes Fridjonsson, et al. [10]. Fingas and Fieldhouse [11] investigated that the mixture of W/O classified into four states: stable, mesostable, unstable, and entrained water. Stable emulsions are brown in appearance and contain 60 to $80 \%$ of water. Mesostable emulsions are founded brown or black and have properties are between stable and unstable emulsions such as $\mathrm{O} / \mathrm{W}$ emulsions. Unstable emulsions are the emulsions that rapidly separate into two phases' water and oil in a short time. Finally, the entrained water is black in appearance and has 30 to $40 \%$ of water content for a few hours. Eventually, it will remain about $10 \%$ in a period of one week. Among these classes only stable and mesostable are considered different from the two other states and they can be characterized as an emulsion.

\subsection{Oil-In-Water Emulsions $(O / W)$}

An oil-in-water emulsion is the emulsion in which the oil exists as the dispersed phase and water as the dispersion medium or continuous phase as shown in Figure 3. In the petroleum industry, the emulsion of $\mathrm{W} / \mathrm{O}$ or $\mathrm{O} / \mathrm{W}$ both can cause huge financial losses if not treated properly [12]. However, the emulsions of $\mathrm{W} / \mathrm{O}$ are more common than $\mathrm{O} / \mathrm{W}$, thus $\mathrm{O} / \mathrm{W}$ emulsions are often pointed as reverse emulsions.

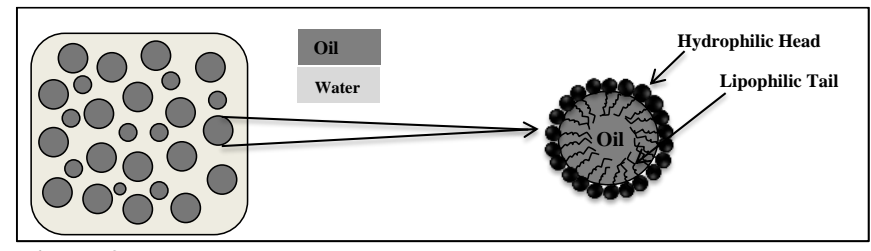

Figure-3.

$\mathrm{O} / \mathrm{W}$ emulsion and structure of emulsifiers on the surface of the dispersed phase (Oil). 


\subsection{Multiple Emulsions}

Multiple emulsions such as water-in-oil-in-water (W/O/W) and oil-in-water-in-oil (O/W/O) can be found in some cases. Usually, multiple emulsions are stabilized using a combination of hydrophilic and hydrophobic surfactants. The Multiple emulsions are more complex and contain very small droplets suspended in larger droplets that are also dispersed in a continuous phase. For instance, the emulsions of W/O/W consist of water droplets entrapped in bigger oil droplets that are sequentially suspended in a continuous water phase as shown in Fig.1. Moreover, these emulsions require at least two emulsifiers to be presented in the system, one emulsifier should has a low (HLB), where another should own a high HLB [13-16].

\section{Emulsion Formation}

The process of emulsion formation is called "emulsification". Emulsification is a dynamic and non-spontaneous process, and energy is required to produce the droplets. The emulsification process usually requires a considerable amount of mechanical energy, to disperse one of the liquids in the form of small droplets in the continuous phase. There are several methods of emulsification including; simple shaking, mixing with rotor-stator systems, liquid injection through porous membranes, or high pressure homogenizers and ultrasound generators. To break up a droplet into smaller sizes, the form of the droplet must be changed and this deformation occurs during the shearing or stirring of the emulsion [17, 18]. The process of droplet deformation and breakup is shown in Figure 4.

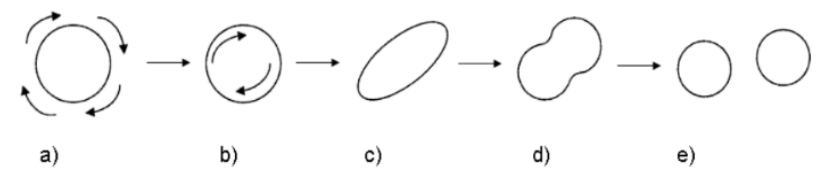

Figure-4.

Droplet deformation during the emulsification process [19].

The main problem in production of crude oil is the formation of an emulsion stabilized by heavy crude oil components such as asphaltenes, resins, and waxes. In the oil field the most common emulsion is the W/O type, and these emulsions are very stable due to the existence of natural surfactants such as asphaltenes, and resins [20]. For the formation of an emulsion three conditions are necessary:

- Immiscibility between phases of the emulsion

- Agitation to disperse one liquid in another

- Existence of surfactants

The emulsion characteristics are changeable from the beginning of the formation to their full resolution, and they depend on various factors such as temperature, agitation speed, time, and pressure. To form a stable emulsion, an emulsifying agent must be added to the system.

\section{Stability of Emulsion}

The emulsion stability related to the type and amount of surfactants. These surfactants promote the stability of the emulsion by forming films around the water drops in water/oil interfaces. The formation of films increase the stability of the emulsion by increasing interfacial viscosity and decreasing interfacial tension (IFT) [21]. It has been investigated that the parameters such as water concentration, mixing speed, and temperature can affect to the emulsion stability [22, 23]. By adding energy to the system the particles will break down into smaller sizes and finally the emulsion becomes more stable which will not be easy to treat.

The physical characteristic of oil/water interfacial films can be changed by temperature, and the emulsion stability can be affected by surfactant solubility in greasy and aqueous phase. Furthermore, by increasing the temperature reduction can be seen in emulsion viscosity, especially in the oily phase of the emulsion [1] Emulsions are thermodynamically unstable and the properties of emulsions are changeable in a certain period of time. Therefore, in terms of better understanding related to mechanism of emulsion stability it is very important to discuss about the thermodynamic and kinetic stability of the emulsions.

\subsection{Thermodynamic and Kinetic Stability of the Emulsions}

Thermodynamic describes about the process which occurs during emulsification or after homogenization. Mehta and Kaur [24] claimed that in thermodynamic approach the stability or instability of the emulsion is related to emulsion droplet size. Thermodynamically, an emulsion is unstable system due to the undesirable contact between oil and water molecules, and they tend to break over a period of time. Furthermore, for stabilizing these kinds of emulsions the emulsifying agents (surfactants) can be used to promote their stability. To achieve kinetically stabilized emulsions the addition of stabilizing agents are necessary [25]. 


\section{Mechanisms of Emulsion Stabilization}

Emulsions are found in various industries, from food and pharmaceuticals to petroleum production and refining. The emulsion stability results from the existence of interfacial barrier preventing coalescence of the dispersed water droplets. Generally, the emulsion stability is influenced by the interface film and mechanism of surfactant adsorption. There are four mechanisms by which emulsions are stabilized. 1) electro- static repulsion; (2) steric repulsion; (3) the Marangoni- Gibbs effect; and (4) thin film stabilization [21]. The following subsections discuss about these four mechanisms.

\subsection{Electrostatic Repulsion}

Electrostatic force results from the interaction between the electrical double layers around the charged droplets which tend to prevent droplet contact (Figure 5). This mechanism occurs by adsorption of an ionic surfactant [26]. However, electrostatic repulsion does not play an important role in the stabilization of water-in-oil emulsions, because of the low dielectric constant of the continuous phase. The emulsion stability is not due to the electrostatic forces, but it is because of the strength of the interfacial film surrounding the dispersed droplets [21].

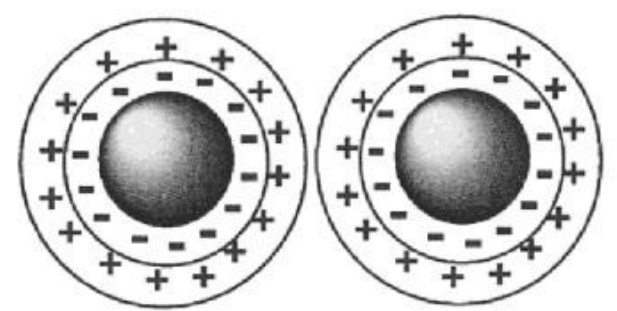

Figure-5.

Schematic representation of electrostatic stabilization [27].

\subsection{Steric Repulsion}

Steric stabilization is generally found in the systems stabilized by non-ionic surfactants and polymers [28]. In this mechanism, the dispersed water droplets will be covered by surfactant molecules and the surfactant tail adsorbed on the surface of the particles prevents droplets to have a close contact as shown in Figure 6. Mainly, this kind of stabilization is responsible for the stabilization of W/O emulsions, since these types of stabilizer contain a non-polar part with strong affinity to oil, and a polar part with a strong affinity to the aqueous phase or water [29].

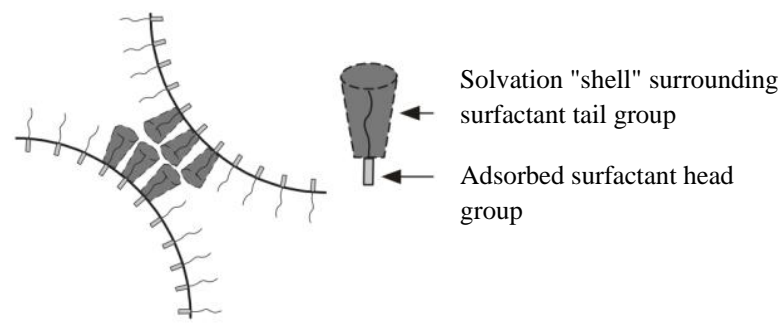

Figure-6.

Schematic representing steric repulsion [30].

\subsection{Marangoni-Gibbs Effect}

The Marangoni-Gibbs effect can stabilize emulsions by preventing the drainage of the continuous phase from between two opposing droplets. This effect occurs due to the deformation of the droplet surface area as they get close along together. When the droplets approach together they make a parallel surface and the film layer attempts to drain as displayed in Figure 7. Moreover, in this phenomena the mechanism of surfactant adsorption and interfacial film are responsible for the stability of the emulsion [31].

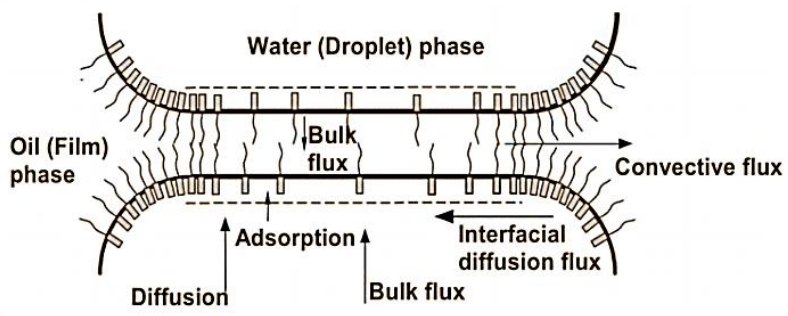

Figure-7.

Emulsion stability according to the Marangoni-Gibbs effect [30]. 


\subsection{Thin Film Stabilization}

In this step, the formation of a rigid and viscoelastic film surrounds the water droplets and prevents the droplets from coalescence [32]. This process can be quite complex and depends on asphaltene chemistry, solvency and the kinetics of diffusion and adsorption [30].

\section{Rheological Study of Emulsions}

\subsection{Viscosity}

Viscosity can be defined as a measure of the internal resistance to flow, or the flow properties of the oil. The most common unit of viscosity is dyne second per square centimeter [dyne s/cm2] which is also called "Poise". In the SI system, units are Pascal.second "Pa.s" or milliPascal seconds "mPa.s" since one mPas.s is equal to one centipoise "cP" in American Society for Testing and Materials "ASTM" standards [33]. It is well known that there are several factors that can influence on the rheological properties of an emulsion, such as water volume fraction, temperature, shear stress, and shear rate, since viscosity is the most measured properties in rheological studies of an emulsion.

According to Newton's law of viscosity, the shear stress is proportional to the shear strain (shear rate). If the viscosity of the fluid does not depend on the shear rate is called "Newtonian fluids" and mostly dilute emulsions show this behavior. However, if the fluid's viscosity depends on the shear rate and have a constant value it called "Non-Newtonian fluid" [34, 35]. Moreover, the rheological behavior of an emulsion can be either Newtonian or non-Newtonian depending upon emulsion [36-38]. Mathematically, Newton's law is expressed in Equation 1.

$$
\eta=\frac{\tau}{\gamma}
$$

Where, $\eta$ is the viscosity, $\tau$ is the shear stress, and $\gamma$ is the shear rate.

Generally, the dynamic viscosity of the oil is varied from about $100 \mathrm{mPa}$.s to almost $105 \mathrm{mPa} . \mathrm{s}$ at room temperature [39]. Viscosity is one of the most important heating oil characteristics and it indicates the flow rate of the oil in fuel systems. Since the heavier oils have high viscosity the determination of this property has taken a particular role in petroleum industries. It is well known that the viscosity of the heavy oils can be increased by reducing the temperature. Therefore, the viscous oils can create many problems throughout the system, and may cause difficulties during pumping and transportation as well [40].

\subsection{Droplet Size}

An emulsion is a dispersion of a liquid (as droplets) inside another immiscible liquid. The size of the water droplets dispersed in the emulsion is an important quantity, as it can affect both the stability and the rheology of the emulsion. Emulsions can also be classified according to the size of the droplets. The droplet size of emulsions depends on some factors such as procedure of emulsification, mixing speed, surfactant type and concentration. Petroleum emulsions have a droplet size in the range of macro and micro. The macro and micro-emulsions can be found in both water-in-oil and oil-inwater emulsion types [41-43]. Figure 8 shows the droplet size of a W/O emulsion.

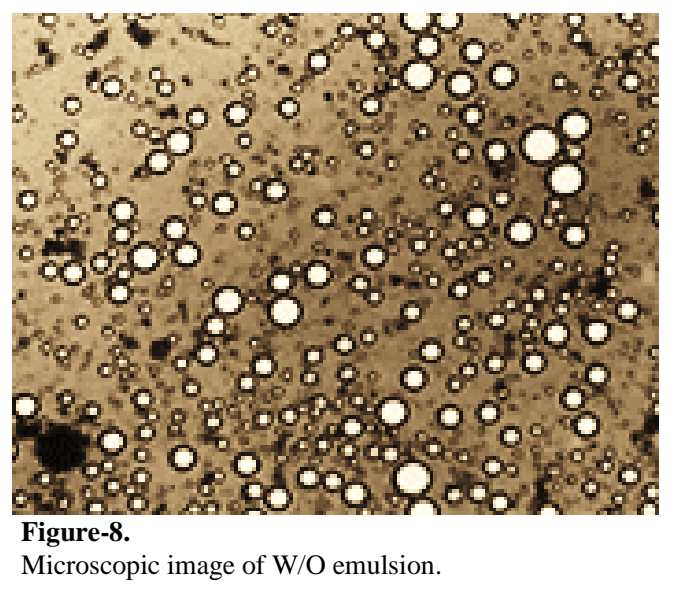

The droplet size and droplet size distribution are important for stability and viscosity properties of the emulsion. In kinetic approach, those emulsions are stable when the number of droplet, droplet size distribution, and order of the droplet do not change significantly over the period of storage [44]. Moreover, increasing the concentration of the emulsifier can reduce the droplet size of emulsion, which could enhance the emulsion stability.

In the case of droplet size, if the concentration of emulsifier is low, the ability of covering the droplets is low as well, thereby the droplets are likely to coalesce with their neighbors and form larger droplets. Surface active materials play an important role in stability of water-in-oil emulsion. The function of emulsifiers is to lower the interfacial tension between water and oil, and to form a cohesive interfacial film around the droplets hence preventing the coalescence of water droplets which finally leads to a stable emulsion [45]. In addition, the droplet size distribution depends on the stirring speed 
and volume of the dispersed phase (water). As the amount of water increases the droplet size and intensity of the distribution increase as well [19].

\section{Conclusions}

Emulsions are considered very important in everyday life. A huge number of products from food to cosmetics deal with emulsions such as mayonnaise, milk, butter, creams, shampoos and many other daily products. This review focused on the study of emulsion types, stability mechanisms and rheology. Based on the reviewed articles, it was found that emulsions stability is related to many factors such as phase ratios, surfactant concentrations, agitation, temperature, and other existed compounds in the system. It is also found that in a stabilized emulsion by a surfactant, the surfactant monomers surround the droplets like a thin film and prevents them from the mechanism of coalescence.

\section{References}

[1] E. Chrisman, V. Lima, and P. Menechini, "Crude oil emulsion-composition stability and characterization," InTech, 3rd ed., InTech, Janeza Trdine, vol. 9, pp. 1-240, 2012.

[2] T. Ichikawa, "Electrical demulsification of oil-in-water emulsion," Colloids and Surfaces A: Physicochemical and Engineering Aspects, vol. 302, pp. 581-586, 2007. Available at: https://doi.org/10.1016/j.colsurfa.2007.03.036.

[3] R. Pal, "Novel shear modulus equations for concentrated emulsions of two immiscible elastic liquids with interfacial tension," Journal of Non-Newtonian Fluid Mechanics, vol. 105, pp. 21-33, 2002. Available at: https://doi.org/10.1016/s03770257(02)00058-7.

[4] H. Zhu and Z. Guo, "Understanding the separations of oil/water mixtures from immiscible to emulsions on super-wettable surfaces," Journal of Bionic Engineering, vol. 13, pp. 1-29, 2016. Available at: https://doi.org/10.1016/s1672-6529(14)601566.

[5] V. Hoshyargar, A. Marjani, F. Fadaei, and S. Shirazian, "Prediction of flow behavior of crude oil-in-water emulsion through the pipe by using rheological properties," Oriental Journal of Chemistry, vol. 28, p. 109, 2012. Available at: https://doi.org/10.13005/ojc/280116.

[6] I. H. Auflem, "Influence of asphaltene aggregation and pressure on crude oil emulsion stability," Doctoral Dissertation, Norwegian University of Science and Technology Trondheim, 2002.

[7] J. T. Foley, P. Forest, and R. H. Rogers, "United States patent 0," 1966

[8] M. Ali and M. Alqam, "The role of asphaltenes, resins and other solids in the stabilization of water in oil emulsions and its effects on oil production in Saudi oil fields," Fuel, vol. 79, pp. 1309-1316, 2000. Available at: https://doi.org/10.1016/s00162361(99)00268-9.

[9] B. B. Filippini, J. John, and A. Deborah, "Water-in-oil-emulsion, US Patent 2004/0176263 A1," 2004.

[10] E. O. Fridjonsson, B. F. Graham, M. Akhfash, E. F. May, and M. L. Johns, "Optimized droplet sizing of water-in-crude oil emulsions using nuclear magnetic resonance," Energy \& Fuels, vol. 28, pp. 1756-1764, 2014. Available at: https://doi.org/10.1021/ef402117k.

[11] M. Fingas and B. Fieldhouse, "Formation of water-in-oil emulsions and application to oil spill modelling," Journal of Hazardous Materials, vol. 107, pp. 37-50, 2004. Available at: https://doi.org/10.1016/j.jhazmat.2003.11.008.

[12] M. E.-S. Abdel-Raouf, Factors affecting the stability of crude oil emulsions. Crude oil emulsions-composition, stability and characterization. Croatia: Intech, 2012.

[13] K. A. Yaqoob, S. Talegaonkar, Z. Iqbal, F. Jalees Ahmed, and R. Krishan Khar, "Multiple emulsions: An overview," Current Drug Delivery, vol. 3, pp. 429-443, 2006. Available at: https://doi.org/10.2174/156720106778559056.

[14] G. Muschiolik, "Multiple emulsions for food use," Current Opinion in Colloid \& Interface Science, vol. 12, pp. 213-220, 2007. Available at: https://doi.org/10.1016/j.cocis.2007.07.006.

[15] R. Pal, "Rheology of simple and multiple emulsions," Current Opinion in Colloid \& Interface Science, vol. 16, pp. 41-60, 2011. Available at: https://doi.org/10.1016/j.cocis.2010.10.001.

[16] J. Jiao and D. J. Burgess, "Rheology and stability of water-in-oil-in-water multiple emulsions containing Span 83 and Tween 80," Aaps Pharmsci, vol. 5, pp. 62-73, 2003. Available at: https://doi.org/10.1208/ps050107.

[17] D. Langevin, S. Poteau, I. Hénaut, and J. F. Argillier, "Crude oil emulsion properties and their application to heavy oil transportation," Oil \& Gas Science and Technology, vol. 59 pp. 511-521, 2004. Available at: https://doi.org/10.2516/ogst:2004036.

[18] T. F. Tadros, Emulsion formation and stability: John Wiley \& Sons, 2013.

[19] M. Lefsaker, "Characterization of alkyd emulsions: Characterization of phase inversion and emulsification properties pre-and post-inversion," Master's Thesis, Department of Chemical Process Technology, 2013.

[20] C. I. Oseghale, E. J. Akpabio, and G. Udottong, "Breaking of oil -water emulsion for the improvement of oil recovery operations in the Niger Delta Oilfields," International Journal of Engineering \& Technology, vol. 2, pp. 1854-1860, 2012.

[21] A. P. Sullivan and P. K. Kilpatrick, "The effects of inorganic solid particles on water and crude oil emulsion stability," Industrial \& Engineering Chemistry Research, vol. 41, pp. 3389-3404, 2002. Available at: https://doi.org/10.1021/ie010927n.

[22] H. N. Abdurahman, M. Y. Rosli, and Z. Jemaat, "Study on demulsification of water-in-oil emulsions via microwave heating technology," Journal of Applied Sciences, vol. 6, pp. 2060-2066, 2006. Available at: https://doi.org/10.3923/jas.2006.2060.2066.

[23] R. M. Abd, A. H. Nour, and A. Z. Sulaiman, "Kinetic stability and rheology of water-in-crude oil emulsion stabilized by cocamide at different water volume fractions," International Journal of Chemical Engineering and Applications, vol. 5, pp. 204-209, 2014. Available at: https://doi.org/10.7763/ijcea.2014.v5.379.

[24] S. Mehta and G. Kaur, "Microemulsions: Thermodynamic and dynamic properties," Thermodynamics, pp. 381-406, 2011. Available at: https://doi.org/10.5772/12954.

[25] R. Pichot, F. Spyropoulos, and I. Norton, "O/W emulsions stabilised by both low molecular weight surfactants and colloidal particles: The effect of surfactant type and concentration," Journal of Colloid and Interface Science, vol. 352, pp. 128-135, 2010 . 
[26] S. N. Molnes, "Physical properties of gelatin based solid emulsions: Effects on drug release in the GI tract," Master's Thesis, Department of Biotechnology, 2013.

[27] Y. Liu, D. J. Sellmyer, and S. Daisuke, Handbook of advanced magnetic materials, illustrate: Springer Science \& Business Media, 2008

[28] Y. Zhang, "The fabrication and application of semi-crystalline and thermoset-thermoplastic composite colloidal particles with well-defined microstructures," Doctoral Dissertation, Johannes Gutenberg-Universität Mainz, 2013.

[29] A. L. B. André, "Investigation of the stability and separation of water-in-oil emulsion," Doctoral Dissertation, Stellenbosch: University of Stellenbosch, 2009.

[30] P. M. Spiecker, The impact of asphaltene chemistry and solvation on emulsion and interfacial film formation: North Carolina State University, 2001.

[31] A. Mosayebi and R. Abedini, "Using demulsifiers for phase breaking of water/oil emulsion," Petroleum \& Coal, vol. 55, pp. 26-30, 2013.

[32] E. J. Ekott and E. Akpabio, "A review of water-in-crude oil emulsion stability, destabilization and interfacial rheology," Journal of Engineering and Applied Sciences, vol. 5, pp. 447-452, 2010. Available at: https://doi.org/10.3923/jeasci.2010.447.452.

[33] I. Gibson, D. Rosen, and B. Stucker, Additive manufacturing technologies: 3D printing, rapid prototyping, and direct digital manufacturing, 2nd ed.: Springer, 2014.

[34] H. M. Lee, J. W. Lee, and O. O. Park, "Rheology and dynamics of water-in-oil emulsions under steady and dynamic shear flow," Journal of Colloid and Interface Science, vol. 185, pp. 297-305, 1997. Available at: https://doi.org/10.1006/jcis.1996.4592.

[35] M. T. Ghannam, B. Abu-Jdayil, and N. Esmail, "Experimental investigation of crude oil-xanthan emulsions flow behavior," Journal of Petroleum Science Research, vol. 3, pp. 1-15, 2014. Available at: https://doi.org/10.14355/jpsr.2014.0301.01.

[36] N. Aomari, R. Gaudu, F. Cabioc'h, and A. Omari, "Rheology of water in crude oil emulsions," Colloids and Surfaces A: Physicochemical and Engineering Aspects, vol. 139, pp. 13-20, 1998.

[37] S. R. Derkach, "Rheology of emulsions," Advances in Colloid and Interface Science, vol. 151, pp. 1-23, 2009.

[38] J. A. Boxall, C. A. Koh, E. D. Sloan, A. K. Sum, and D. T. Wu, "Measurement and calibration of droplet size distributions in water-in-oil emulsions by particle video microscope and a focused beam reflectance method," Industrial \& Engineering Chemistry Research, vol. 49, pp. 1412-1418, 2010. Available at: https://doi.org/10.1021/ie901228e.

[39] M. Fingas and B. Fieldhouse, "Water-in-oil emulsions: formation and prediction," Handbook of Oil Spill Science and Technology, vol. 225, 2014.

[40] S. J. Rand, Significance of tests for petroleum products A. W. Verstuyft (Ed.). Newburyport: ASTM International, 2010.

[41] D. Balzer, "Microemulsion cleaning compositions containing surfactant," pp. 1-4, 1999.

[42] M. J. Lawrence and G. D. Rees, "Microemulsion-based media as novel drug delivery systems," Advanced Drug Delivery Reviews, vol. 45, pp. 89-121, 2000. Available at: https://doi.org/10.1016/s0169-409x(00)00103-4.

[43] E. J. Windhab, M. Dressler, K. Feigl, P. Fischer, and D. Megias-Alguacil, "Emulsion processing-from single-drop deformation to design of complex processes and products," Chemical Engineering Science, vol. 60, pp. 2101-2113, 2005. Available at: https://doi.org/10.1016/j.ces.2004.12.003.

[44] M. Sanchez, M. Berjano, A. Guerrero, and C. Gallegos, "Emulsification rheokinetics of nonionic surfactant-stabilized oil-inwater emulsions," Langmuir, vol. 17, pp. 5410-5416, 2001. Available at: https://doi.org/10.1021/la000723w.

[45] S. Akbari, N. H. Abdurahman, and F. Fayaz, "The influence of process parameters on stability of water-in-crude oil emulsion stabilized by span 80," International Journal of Engineering, Science and Technology, vol. 4 pp. 526-534, 2015. 